\title{
Influence of a laser profile in impedance mismatch techniques applied to carbon EOS measurement
}

\author{
A. Aliverdiev ${ }^{1,2}$, D. Batani ${ }^{3}$, and R. Dezulian ${ }^{4}$ \\ ${ }^{1}$ Institute for Geothermal Researches DSC of Russian Academy of Sciences - Pr. Shamilya 39A, 367030, Makhachkala, Russia \\ ${ }^{2}$ Dagestan State University, Gadjieva Str. 43A, 367025, Makhachkala, Russia \\ ${ }^{3}$ University Bordeaux, CEA, CNRS, CELIA (Centre Laser Intense et Applications), UMR 5107, 33405 Talence, France \\ ${ }^{4}$ Dipartimento di Fisica 'G. Occhialini', Università di Milano-Bicocca, Milano, Italia \\ (Received 15 April 2013; revised 30 May 2013; accepted 4 June 2013)
}

\begin{abstract}
We present a recent numerical analysis of impedance mismatch technique applied to carbon equation of state measurements. We consider high-power laser pulses with a Gaussian temporal profile of different durations. We show that for the laser intensity $\left(\approx 10^{14} \mathrm{~W} / \mathrm{cm}^{2}\right)$ and the target design considered in this paper we need to have laser pulses with rise-time less than $150 \mathrm{ps}$.
\end{abstract}

Keywords: equation of state (EOS), high pressures, impedance mismatch technique

\section{Introduction}

The equation of state (EOS) of carbon at high pressures is a subject of interest for several branches of science, including astrophysics, material science, and applied engineering (first of all including fusion research). In particular, a very important point (in particular for the explanation of large magnetic fields of giant planets such as Uranus and Neptune) is the existence of a metallic phase of carbon ${ }^{[1-4]}$. To reach it in laboratory conditions, it is possible to use laser-driven shocks. The pressure (in Mbar) of such shocks can be estimated by $P=11.6\left(I / 10^{14}\right)^{3 / 4} \lambda^{-1 / 4}(A / 2 Z)^{7 / 16}$ $\left(Z^{*} t / 3.5\right)^{-1 / 8}$, where $I$ is the laser intensity on the target in $\mathrm{W} / \mathrm{cm}^{2}, \lambda$ is the laser wavelength in $\mu \mathrm{m}$, and $A, Z$, and $Z^{*}$, respectively, are the mass number, the atomic number, and the effective ionization degree of the target, and the time $t$ is in $\mathrm{ns}^{[5]}$. So intensities of the order of $10^{14} \mathrm{~W} / \mathrm{cm}^{2}$, which can be obtained quite easily, allow getting pressures of the order of 10 Mbar.

A well-known experimental method to measure the EOS is based on the impedance mismatch technique and consists in measuring the shock velocity of two different materials (test and reference) at the same time. The shock-wave measurements are realized by a streak camera recording the emission from the rear side of the shocked target. Using time-resolved imaging, we can experimentally determine the times of the shock arrival for each part of target

Correspondence to: A. Aliverdiev, Institute for Geothermal Researches DSC of Russian Academy of Sciences, Pr. Shamilya 39A, 367030, Makhachkala, Russia. Email: aliverdi@mail.ru (see Figure 1), and afterwards the velocity of the shock propagating through the two steps, $D_{\mathrm{Al}}$ and $D_{\mathrm{C}}$. Then if the EOS (and hence the shock adiabat) of the base material (aluminium) is known, we can calculate an EOS point for the test material (carbon) as the intersection in the $(P, U)$ plane of the line $P=\rho_{\mathrm{C}} D_{\mathrm{C}} U$ (momentum conservation law; $\rho_{\mathrm{C}}$ is the density of cold carbon) with the reflected isentropic release drawn from the intersection of $P=\rho_{\mathrm{Al}} D_{\mathrm{Al}} U\left(\rho_{\mathrm{Al}}\right.$ is the density of cold aluminium) with the aluminium Hugoniot adiabat (see Figure 2) ${ }^{[6,7]}$.

One of the critical points of this method is the effect of the temporal profile of the high-power pulse. Indeed, an ideal shock can be produced only with a high-power flattop laser pulse, and the analysis of the effect of the real temporal profile of a high-power laser in experiments is important, because it can be a substantial contribution to the total experimental error. The aim of the present work was to realize a set of simulations for Gaussian pulses with different durations and to analyse the effect on the calculation of shock velocities.

\section{Simulations}

For the realization of simulations we have used the hydrocode MULTI (multigroup radiation transport in multilayer foils $)^{[8]}$. We have used the SESAME equation of state for aluminium ${ }^{[9]}$ and porous carbon EOS calculated by MPQEOS ${ }^{[10]}$ with a reduced initial density $\left(1.6 \mathrm{~g} / \mathrm{cm}^{3}\right)^{[11,12]}$, as presented in Ref. Ref. [13]. For each 

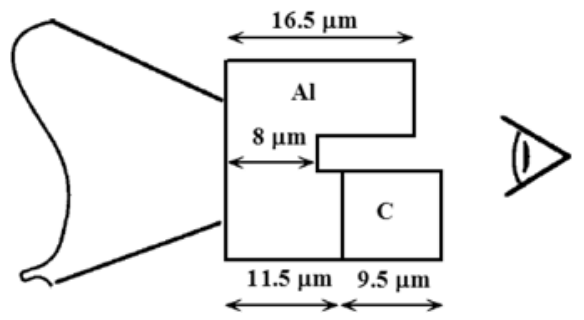

Figure 1. Sketch of the configuration of EOS measurements using the impedance mismatch technique. A streak camera measures the times of the shock arrival; the shock velocities $D_{\mathrm{Al}}$ and $D_{\mathrm{C}}$ are calculated from the difference of these times.

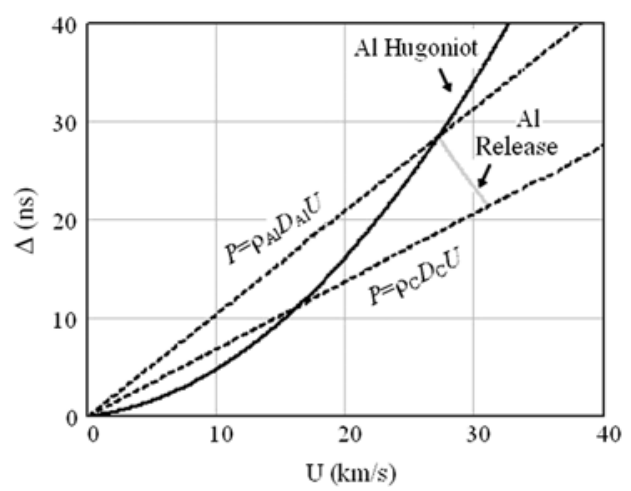

Figure 2. Calculation of the $(P, U)$ EOS point for carbon from measured $D_{\mathrm{Al}}, D_{\mathrm{C}}$ and $\mathrm{Al}$ Hugoniot adiabat using the impedance mismatch method.

case we have realized three different 1D 'sub-simulations' for the three parts of the target: (i) Al $8 \mu \mathrm{m}$, (ii) Al $16.5 \mu \mathrm{m}$, and (iii) $\mathrm{Al}-\mathrm{C} 11.5 \mu \mathrm{m}+9.5 \mu \mathrm{m}$ corresponding to $\mathrm{Al}$ base, Al step, and carbon step (see Figure 1), and have determined the shock arrival times to the rear target surface, as in the real experiment $^{[13]}$.

We have tested four Gaussian laser profiles with the same peak intensity $\left(I_{\max }=2.05 \times 10^{14} \mathrm{~W} / \mathrm{cm}^{2}\right)$ and full width at half maximum (FWHM) durations $\tau=200,300,450$, and 600 ps. Gaussian profiles were calculated by $I=$ $I_{\max } \exp \left(-2.77\left(\frac{t-\tau}{\tau}\right)^{2}\right)$ (see Figure 3 ). So the initial intensity at zero time was $6.3 \%$ of the maximum. Also we used an ideal flat-top pulse of the same intensity and 450 ps duration for reference.

\section{Results and discussion}

Figure 4 presents the dependence of the difference between shock arrivals for Gaussian pulses and the reference one (flat top) as a function of duration $\tau$ for the three parts of the target.

If the rise-time of the Gaussian pulse is small $(\tau \leqslant 300 \mathrm{ps}$ from Figure 4), the difference is the same for the base and both steps. This implies that the shock velocity calculations are not affected. Physically this means that for $\tau \leqslant 300$ ps the shock becomes stationary both in the base ( $8 \mu \mathrm{m}$ thick) and in the steps. In this case the effect of the Gaussian pulses is just to shift all breakout times by the same amount. We

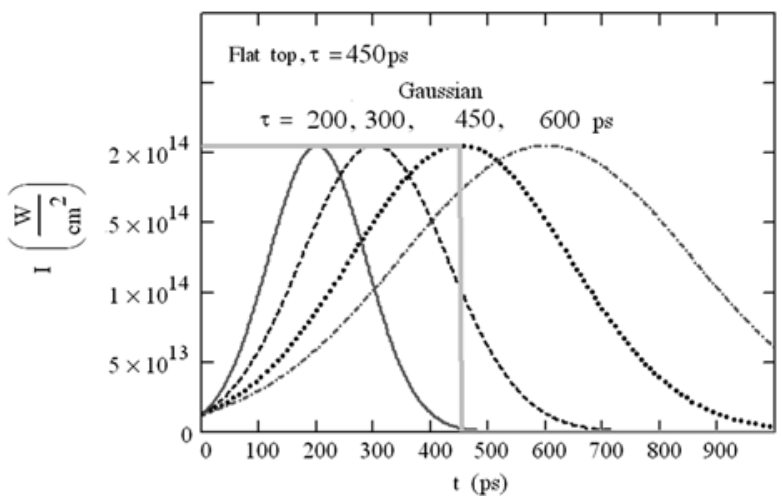

Figure 3. Laser pulse profiles used in the simulations.

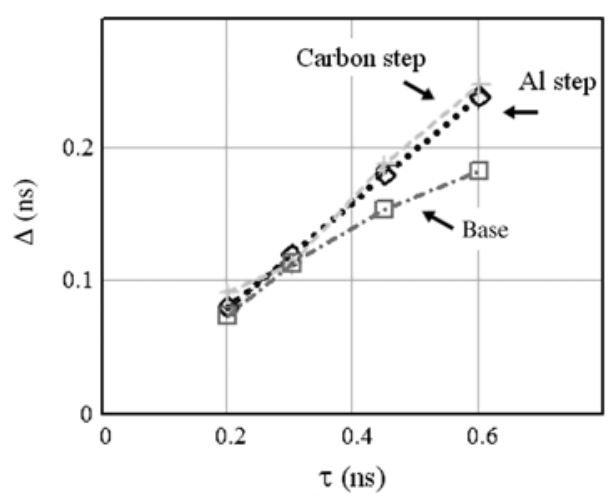

Figure 4. The dependences of the difference between shock arrivals for Gaussian pulses and the reference one (flat top) from $\tau$ for all three parts of the target: (i) base (Al: $8 \mu \mathrm{m}$ ); (ii) Al step (Al: $16.5 \mu \mathrm{m}$ ); and (iii) carbon step (Al-C: $11.5 \mu \mathrm{m}+9.5 \mu \mathrm{m})-\Delta_{\text {base }}, \Delta_{\mathrm{Al}}$ and $\Delta_{\mathrm{C}}$, correspondingly. All dependences are very close to each other for $\tau \leqslant 300$ ps.

notice that these spatial profiles are not exactly the same (see Figure 5), but the same time differences for the shock breakouts are enough for a correct calculation of shock velocities.

Simulations done for $\tau>300$ ps show that instead the shock has the time to become stationary in the steps (the difference is indeed the same for the two steps) but not for base. In this case the shock velocity cannot simply be calculated as $D=$ thickness $/\left(t_{\text {step }}-t_{\text {base }}\right)$.

\section{Conclusion}

On the basis of hydro simulations, we can conclude that, with the considered laser intensity $\left(\approx 10^{14} \mathrm{~W} / \mathrm{cm}^{2}\right)$ and target design, it is necessary to have the duration of the Gaussian pulse not longer 300 ps. In the opposite case, the shock does not have the time to become stationary before the shock breaks out of the base. These results can be extrapolated to pulses with any shape by saying that the pulse rise-time should be less than 150 ps. Depending on target thickness, the pulse rise-time required will be different; however, the method described here is general. 


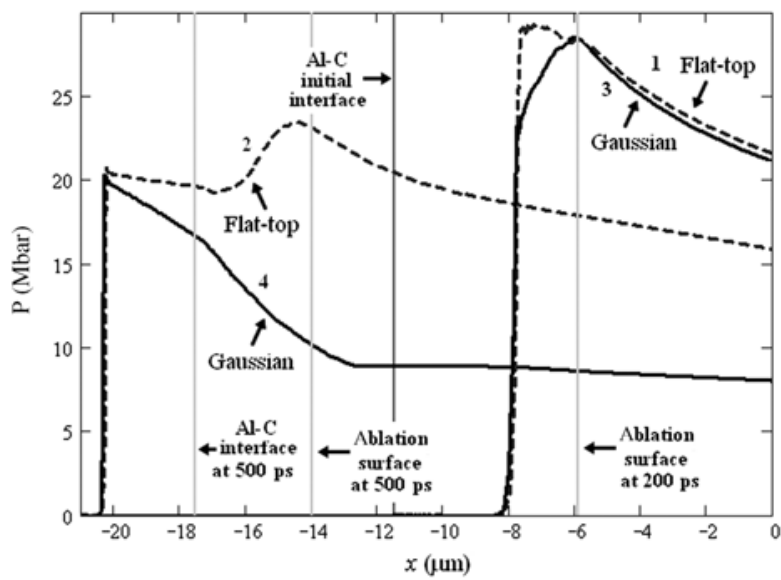

Figure 5. Comparison of spatial profiles for the shocks initiated by flattop and Gaussian (FWHM duration 300 ps) pulses. Lines 1 and 2 (dashed) are shock profiles for a flat-top laser pulse at 200 and $500 \mathrm{ps}$ (close to the shock breakout on the base and the carbon step). Lines 3 and 4 (solid) are shock profiles for a Gaussian laser pulse at 314 and 614 ps. We notice that both profiles have the same time shift $\Delta=114 \mathrm{ps}$. Despite the profile differences the fronts for both shocks are very close to each other. The laser strikes from the right. Zero on $x$ corresponds to the target front. The vertical line at $-11.5 \mu \mathrm{m}$ is the initial $\mathrm{Al}-\mathrm{C}$ interface. Ablation surfaces and $\mathrm{Al}-\mathrm{C}$ interfaces for flat-top profiles at 200 and $500 \mathrm{ps}$ are indicated (for $200 \mathrm{ps}$, the Al-C interface is the initial one).

\section{Acknowledgements}

The work was partially supported by ESF (SILMI, 3964), EU COST program, and RFBR (12-01-96500, 11-01-00707).

\section{References}

1. F. P. Bundy, Physica A 156, 169 (1989).

2. M. P. Grumbach, and R. M. Martin, Phys. Rev. B 54, 15730 (1996).

3. M. Van Thiel, and F. H. Ree, Phys. Rev. B 48, 3519 (1993).

4. A. A. Correa, S. A. Bonev, and G. Galli, PNAS 103, 1204 (2006).

5. D. Batani, A. Balducci, W. Nazarov, T. Lower, T. Hall, M. Koenig, B. Faral, A. Benuzzi, and M. Temporal, Phys. Rev. E. 63, 046410 (2001).

6. Ya. B. Zel'dovich, and Yu. P. Raizer, Physics of Shock Waves and High-Temperature Hydrodynamical Phenomena (Dover, New York, 2002).

7. P. M. Celliers, G. W. Collins, D. G. Hicks, and J. H. Eggert, J. Appl. Phys. 98, 113529 (2005).

8. R. Ramis, R. Schmalz, and J. Meyer-ter-Vehn, Comput. Phys. Commun. 49, 475 (1988).

9. K. S. Holian ed. T-4 Handbook of Material Properties Data Bases, Vol. 1C: Equations of State, LANL Report No. LA10160-MS UC-34 (1984).

10. A. Kemp, and J. Meyer-ter-Vehn, Nucl. Instrum. Methods Phys. Res. A. 415, 674 (1998).

11. A. Aliverdiev, D. Batani, R Dezulian, and T. Vinci, Radiat. Eff. Defects Solids 165, 566 (2010).

12. A. Aliverdiev, D. Batani, R Dezulian, and T. Vinci, Nukleonika 56, 165 (2011).

13. D. Batani, F. Strati, H. Stabile, M. Tomasini, G. Lucchini, A. Ravasio, M. Koenig, A. Benuzzi-Mounaix, H. Nishimura, Y. Ochi, J. Ullschmied, J. Skala, B. Kralikova, M. Pfeifer, Ch. Kadlec, T. Mocek, A. Prag, T. Hall, P. Milani, E. Barborini, and P. Piseri, Phys. Rev. Lett. 62, 065503 (2004). 